\title{
Certolizumab Pegol in the Treatment of Psoriasis and Psoriatic Arthritis: Preliminary Real-Life Data
}

\author{
Annunziata Dattola · Maria Vittoria Cannizzaro • Mauro Mazzeo •
}

Luca Bianchi

Received: September 7, 2017 / Published online: November 14, 2017

(c) The Author(s) 2017. This article is an open access publication

\section{ABSTRACT}

Introduction: We present the results of real-life tests conducted in adults affected by psoriatic arthritis (PsA) with mild cutaneous involvement to evaluate the efficacy of certolizumab pegol (CZP), an anti-tumor necrosis factor-alpha agent approved in Europe for the treatment of rheumatoid arthritis and PsA.

Methods: Assessments included an evaluation of the Psoriasis Area and Severity Index (PASI) and the Disease Activity Score computed on 44 joints (DAS-44) correlated to the erythrocyte sedimentation rate (ESR) (DAS44-ESR). A total of 41 patients (16 men, 25 women; mean age $59.8 \pm 8$ years) completed the study. Of these, 36 patients were affected by both PsA and psoriasis, and five patients were affected only by PsA. A total of 32 patients (group A) completed 3 months of treatment (W12), and 12 patients completed 6 months of treatment (W24) (group B).

Enhanced content To view enhanced content for this article go to http://www.medengine.com/Redeem/ 7CCCF0607A3E0482.

Annunziata Dattola and Maria Vittoria Cannizzaro contributed equally to the work.

A. Dattola $(\bowtie) \cdot$ M. V. Cannizzaro - M. Mazzeo .

L. Bianchi

Department of Dermatology, University of Rome

"Tor Vergata", Rome, Italy

e-mail: nancydattola@gmail.com
Results: The clinical efficacy of CZP was consistent on both the cutaneous and rheumatic components of the treatment. The mean PASI score decreased from $4.4 \pm 4.7$ at baseline (BL) to $2.3 \pm 3.7$ at W12 (group A), and from $5.1 \pm 5.7$ at BL to $0.8 \pm 1.2$ at W24 (group B). The DAS44-ESR decreased from $4.4 \pm 0.6$ at BL to a mean of $2.2 \pm 0.9$ at W12 (group A) and from $4.1 \pm 0.6$ at BL to a mean of $1.9 \pm 0.5$ at W24 (group B). No adverse events were reported.

Conclusion: Our results demonstrate that CZP can be used safely and effectively to treat both the cutaneous and joint components of PsA. However, long-term data are needed to confirm our preliminary observations.

Keywords: Anti-TNF- $\alpha$; Biologics therapy; Certolizumab pegol; Psoriasis; Psoriatic arthritis; Real life data

\section{INTRODUCTION}

Psoriatic arthritis (PsA) is a chronic inflammatory arthritis that occurs in patients with skin psoriasis (PsO), with both genders affected equally [1]. The prevalence of $\mathrm{PsO}$ varies from 2 to $3 \%$ of the general population, and PsA affects $10-30 \%$ of patients with PsO [2]. PsA is a heterogeneous disease with various manifestations, including peripheral arthritis, enthesitis, dactylitis, axial arthritis, and skin and nail 
psoriasis. More than half of the patients affected by PsA exhibit progressive erosive arthritis, associated with severe functional impairment [3]. The pathophysiology of PsA remains to be completely elucidated, but the overexpression of tumor necrosis factor- $\alpha$ (TNF- $\alpha$ ) is believed to play a key role in the pathogenic mechanisms linking PsA and PsO [4]. The PsA synovium shows infiltration with $\mathrm{T}$ cells, B cells, and macrophages. Clonally expanded CD8+ T cells are frequently observed in PsA. Plasmacytoid dendritic cells are thought to play a key role in $\mathrm{PsO}$, and there is some evidence that these cells are also involved in PsA. The extensive bone lesions in patients with PsA are consistent with the findings of osteoclastic progenitors in the peripheral blood of these patients, as well as upregulation of the receptor activator of nuclear factor kappa $b(\mathrm{NF}-\mathrm{\kappa B})$ ligand (RANKL) in the synovial lining layer. Cytokines produced by T-helper 17 (Th17) cells are likely to be important in PsA, given their prominence in PsO and in other forms of spondylarthritis [5]. Innate immunity also seems to play a role in the pathogenesis of PsA. Current treatment options for patients with PsA include non-steroidal inflammatory drugs, glucocorticoids, disease-modifying antirheumatic drugs (DMARDs), biologic agents [such as the anti-TNF- $\alpha$ drugs adalimumab, etanercept, golimumab, infliximab, and certolizumab pegol (CZP), and the interleukin (IL)-12/23 inhibitor ustekinumab], and the phosphodiesterase- 4 inhibitor apremilast. To date, biologic agents have been suggested to have a more favorable side effect profile than synthetic DMARDs. Anti-TNF- $\alpha$ has shown efficacy in improving peripheral arthritis and psoriasis symptoms, as well as other PsA manifestations, such as enthesitis, dactylitis, axial disease, physical function, quality of life, productivity, and work disability, and by inhibiting radiographic progression.

CZP is a novel TNF inhibitor formed by a humanized Fab fragment $(50 \mathrm{kDa})$ fused to a $40-\mathrm{kDa}$ polyethylene glycol (PEG) moiety (a nontoxic and nonimmunogenic polymer). The lack of the Fc region in CZP prevents activities such as complement fixation and antibody-mediated cytotoxicity. The attachment of the 40-kDa PEG moiety to the Fab fragment markedly increases the half-life of CZP to a value comparable with that of a whole antibody product $[6,7]$. CZP has been shown to improve patient-reported outcomes in rheumatoid arthritis (RA), PsA, and PsO [8]. The efficacy and safety of CZP as a treatment for PsA were investigated in the RAPID-PsA study [9], which is the first published trial in PsA to include patients with prior exposure to TNF inhibitors. In Europe, CZP was approved in 2009 for the treatment of active PsA in adults with inadequate response to previous DMARD therapy; it has just recently been approved in Italy. The aim of the study reported here was to evaluate the efficacy, safety, and patient-reported outcomes (PROs) of CZP in real life.

\section{METHODS}

This study was conducted at the Department of Dermatology of the University of Rome "Tor Vergata". The study was approved by the local ethics committee in accordance with the ethical principles of the Declaration of Helsinki and was consistent with the guidelines for good clinical practice. All patients provided written consent.

Inclusion criteria were an age of $>18$ years and a clinical and instrumental (ultrasound and/or nuclear magnetic resonance) diagnosis of PsA for $>6$ months in non-responders or those intolerant to DMARDs, including methotrexate (MTX), azathioprine, or leflunomide. The study also included patients previously treated with other biologics (anti-TNF drugs and/or anti-IL12/23). The reasons for discontinuation of prior biologic agents were primary inefficacy, long-term loss of efficacy, or adverse events. Patients participating in the study who had been previously treated with other biologics were asked to have at least a 1-month washout time (period between ending the treatment with the previous biologic drug and starting CZP). Patients with a medical history of malignancy (in the last 5 years) and systemic infections, with a history of active infectious disorders (including active or latent tuberculosis), and/or with a history of chronic or recurrent serious infective diseases, 
opportunistic infections, demyelinating diseases, or live vaccines within 3 months prior to the first infusion were excluded from the study.

\section{Clinical and Laboratory Values}

Clinical and laboratory evaluations were performed at baseline (BL), week 12 (W12), and week 24 (W24) of treatment. Assessment included medical history, a general and dermatologic physical examination, and the computation of the Psoriasis Area and Severity Index (PASI), which is a score used to measure the severity and extent of psoriasis, and the Disease Activity Score computed on 44 joints (DAS-44) correlated to the erythrocyte sedimentation rate (ESR) during the first hour (DAS44-ESR). PROs included the health assessment questionnaire modified for spondyloarthritis (SpA-HAQ) and the quality of life questionnaire [Dermatology Life Quality Index (DLQI)]. The patients were also asked to evaluate the pain on visual analog scale (pain-VAS) ranging from 0 (absence of pain) to 100 (the worst pain). The DAS44 was measured according to American College of Rheumatology (ACR) guidelines by evaluating Ritchie's index, the number of swollen and tender joints, the ESR, and the general health condition. Active rheumatic disease was considered when the DAS44 was $>3.7 ;$ a DAS44-ESR of $\leq 2.4$ was considered to indicate an adequate clinical response, and remission was considered when the DAS44 was $<1.6$. Before enrolment, patients underwent screening tests as suggested by the European guidelines. All patients were screened by laboratory tests (including complete blood cell count, liver and renal function tests, urinalysis, protein electrophoresis, tests for anti-nuclear antibodies and anti-extractable nuclear antigen antibodies, tests for hepatitis A, B, C and human immunodeficiency viruses, pregnancy test, Mantoux skin or QuantiFERON®-TB Gold tests, chest X-ray and electrocardiography). Laboratory values included the ESR and the C-reactive protein (CRP) with a normal range from 0 to $15 \mathrm{~mm} / \mathrm{h}$ and 0 to $0.5 \mathrm{mg} / \mathrm{dl}$, respectively.

\section{Endpoints}

The primary endpoint was an evaluation of the clinical efficacy and of the safety of CZP on joint involvement during treatment according to the DAS44-ESR (see clinical values) and CRP. The secondary endpoint was an evaluation of the reduction of the PASI and pain-VAS. The third endpoint was an evaluation of the PROs assessed using the DLQI and SpA-HAQ. Clinical and laboratory evaluations were performed for both cutaneous and joint diseases at BL and at W12, and W24 of treatment. All patients were treated with CZP 400-mg loading dose at weeks 0, 2, and 4, followed by CZP $200 \mathrm{mg}$ every 14 days.

\section{Statistical Analysis}

Data from the clinical laboratory analyses were entered into a Windows-based database (Excel 2007; Microsoft Corp., Redmond, WA ), and all statistical analyses were performed using GraphPad Prism 5 statistical software (GraphPad Software, San Diego, CA). Results were expressed as the mean \pm standard deviation. The significance of differences in the mean values obtained at BL and at W12 and W24 of treatment was assessed using a paired Student's $t$ test (statistical significance set at $p \leq 0.05$ ).

\section{RESULTS}

Of the 45 patients enrolled in the study, 41 completed it. Our study population included 16 males and 25 females, with a mean age of $59.8 \pm 8$ (range 39-75) years, who were affected by moderate to severe PsA with mild cutaneous involvement. Of these, 36 patients were affected by PsA and plaque-type $\mathrm{PsO}$, and five patients were affected only by PsA. Thirty-two patients (designated group A) completed 3 months of treatment (W12), and 12 patients completed 6 months (W24) (designated group B). Fourteen patients were naïve to anti-TNF- $\alpha$ treatment, and 27 patients had previously received treatment with other TNF- $\alpha$ inhibitors. The most frequent comorbidities seen in our population were hypertension, obesity, and 
Table 1 Demographic data and clinical features of the studied population

\begin{tabular}{|c|c|}
\hline Demographic data and clinical features & Values \\
\hline Number of patients & 41 \\
\hline Age (years) & $59.8 \pm 8$ \\
\hline Male/female & $16 \mathrm{M} / 25 \mathrm{~F}$ \\
\hline Onset of the disease (years) & $\begin{array}{l}13.5 \\
\quad \pm 14.6\end{array}$ \\
\hline Plaque-type psoriasis & $37(90.2 \%)$ \\
\hline Psoriatic arthritis & $41(100 \%)$ \\
\hline $\begin{array}{l}\text { Mean number of previous systemic } \\
\text { treatments }\end{array}$ & $1.7[1-3]$ \\
\hline Mean number of previous biologics & $1.4[1-5]$ \\
\hline \multicolumn{2}{|c|}{$\begin{array}{l}\text { Data are presented as mean } \pm \text { standard deviation, the } \\
\text { mean with the range in square brackets, as a number with/ } \\
\text { without the percentage in parenthesis }\end{array}$} \\
\hline \multicolumn{2}{|c|}{$\begin{array}{l}\text { hyperlipidemia. Patients' demographics and } \\
\text { clinical laboratory characteristics are summa- } \\
\text { rized in Table } 1 \text {. The clinical efficacy was con- } \\
\text { sistent on both the cutaneous and rheumatic } \\
\text { components of the condition in both groups. } \\
\text { The mean PASI score decreased from } 4.4 \pm 4.7 \\
\text { at BL to } 2.3 \pm 3.7 \text { at W12 (group A), and from } \\
5.1 \pm 5.7 \text { at BL to } 0.8 \pm 1.2 \text { at W } 24 \text { (group B). } \\
\text { The DAS } 44 \text {-ESR decreased from } 4.4 \pm 0.6 \text { at BL } \\
\text { to a mean of } 2.2 \pm 0.9 \text { at W12 (group A) and } \\
\text { from } 4.1 \pm 0.6 \text { at BL to a mean of } 1.9 \pm 0.5 \text { at }\end{array}$} \\
\hline
\end{tabular}

W24 (group B) (Figs. 1, 2). The mean pain VAS decreased from 80 at BL to a mean of 35.9 after 12 weeks of treatment. The DLQI and SpA-HAQ scores improved from a mean of 26 to a mean of 8 after 12 weeks, and from a mean of 1.65 at BL to a mean of 0.89 at $\mathrm{W} 12$, respectively (data not shown). The clinical pictures of some of the patients affected by dactylitis and $\mathrm{PsO}$ at $\mathrm{BL}$ and after 12 and 24 weeks of treatment are shown in Figs. 3, 4, 5.

\section{Safety}

Efficacy and safety evaluations were performed every 3 months. Of the 45 patients, three dropped out before completion of the study; one prematurely left the study because treatment with CZP was halted due to the onset of alopecia, and the remaining two patients left the study due to secondary inefficacy of the drug, with the latter patient ultimately undergoing bariatric surgery for complications. Liver and renal functions were monitored in all patients during the treatment, and no patient presented abnormal values. CZP was safe and well tolerated in all patients compared to traditional systemic treatments for PsA.

Our results demonstrate that CZP is a safe and effective treatment for both cutaneous and joint components. No serious infectious diseases that would require hospitalization or
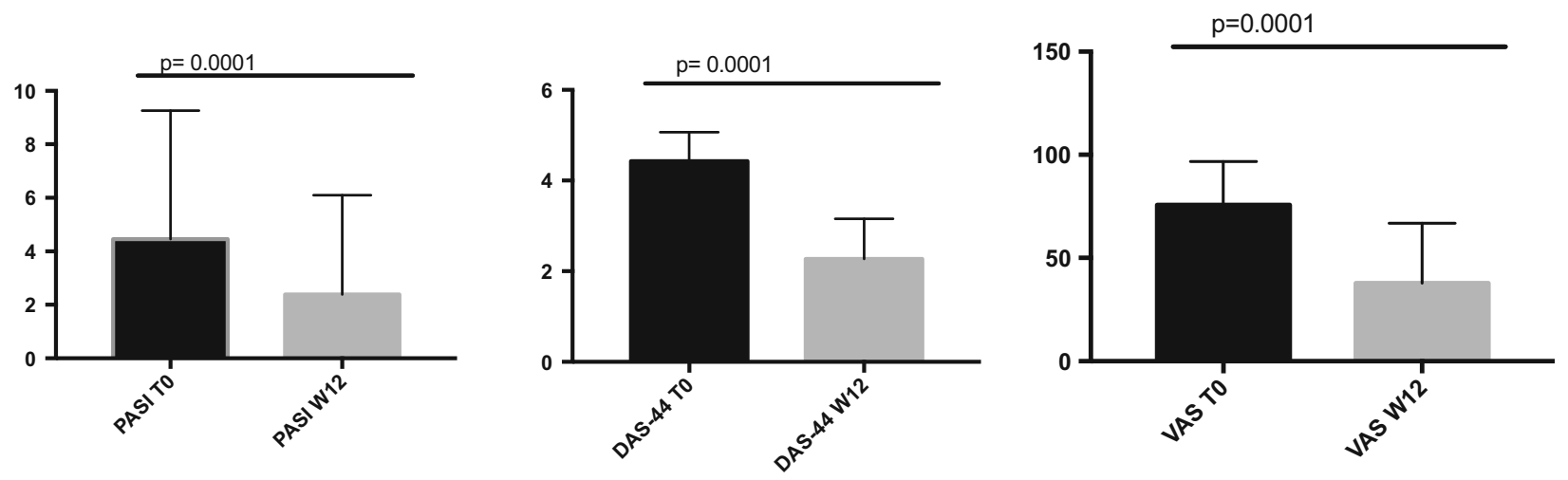

Fig. 1 Comparison of the Psoriasis Area and Severity Index (PASI) score, the Disease Activity Score computed on 44 joints (DAS-44), and the Pain Visual Analog scale
(VAS) score for group A patients (32 patients who completed 3 months of treatment) at baseline (T0) and at 12 weeks of treatment (W12) 

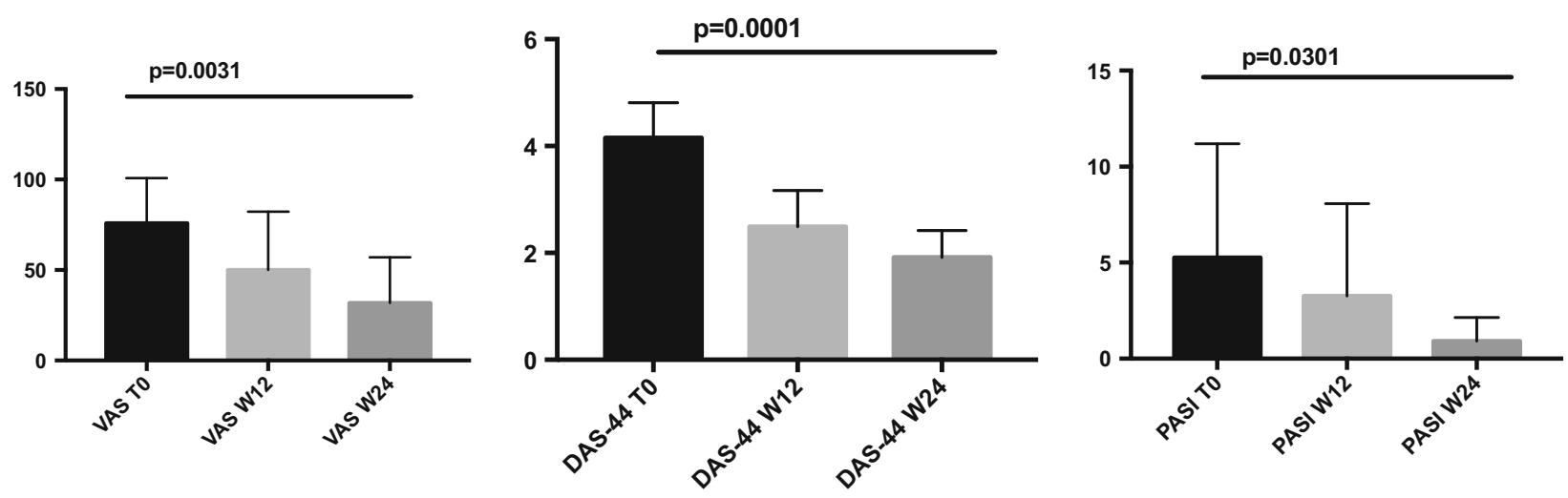

Fig. 2 Comparison of the PASI score, DAS-44, and pain VAS score for group B patients (12 patients who completed 6 months of treatment) at baseline (T0) and after 24 weeks of treatment (W24)

lymphoproliferative disorders (lymphomas) were reported.

\section{DISCUSSION}

The aim of our study was to evaluate the efficacy and safety of CZP in real life and assess the PROs at 12 and 24 weeks of treatment.

Of the five TNF inhibitors currently approved for the treatment of PsA [10] (CZP, adalimumab, etanercept, golimumab and infliximab), CZP is the latest drug to receive approval for the treatment of PsA in the EU and USA [11].

Several controlled clinical trials have demonstrated the efficacy and tolerability of CZP in the treatment of PsA $[9,12,13]$. In the RAPID-PsA trial, CZP effectively improved the signs and symptoms of patients with PsA with a rapid onset of action [differences in clinical measures by week $1(p<0.001)]$ and a statistically significant difference in ACR20 (improvement in RA by $20 \%$ according to ACR criteria) at week 12 (the primary clinical endpoint), which was maintained up to week 24, was observed [9].

Our study emphasizes the efficacy of CZP in association with MTX or in monotherapy. No difference was found in terms of efficacy and safety between naïve and patients who had been previously treated with other TNF- $\alpha$ inhibitors. Although the AIFA (Agenzia Italiana del Farmaco) requires a combined regimen of CZP and MTX, the 41 patients enrolled in the present study were either treated with CZP in a combination regimen ( 8 patients) or as monotherapy (33 patients) because of referred previous intolerance or inefficacy to MTX, without any difference in the achievement of the clinical score, indicating disease remission. The drug was effective in patients who were either naïve and/or previously unresponsive to other anti-TNFs for PsA and PsO.

The statistical analysis of our data showed a significant reduction in the considered indices for assessing joint and cutaneous involvement of the disease, i.e. the SpA-HAQ, DAS44, and PASI.

In patients affected by PsA, improvement of the DAS44-ESR, which decreased from a mean of $4.4 \pm 0.6$ at BL to a mean of $2.2 \pm 0.9$ as early as week 12 of treatment, and reduction of the pain VAS score also at this timepoint were notable; further improvement was seen as the treatment progressed. In our cohort of patients, the majority of adverse events reported were mild and did not require treatment adjustment, corroborating data reported in the literature. The CZP loading dose may contribute to the observed fast response within the first 4 weeks of treatment (data not shown). CZP was effective in terms of improving physical function in the context of dactylitis and enthesitis and demonstrated rapid improvements in skin involvement, as measured by the PASI score. At W24, improvements in nail disease were also 


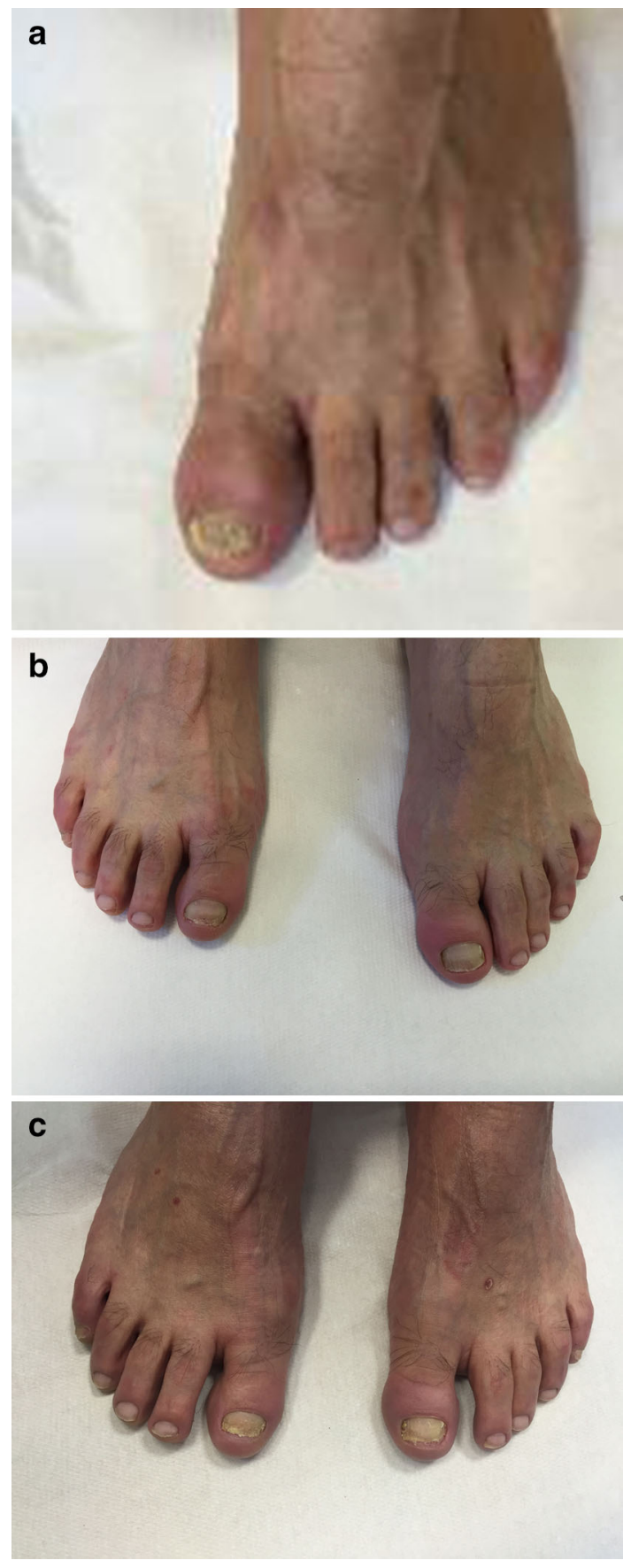

Fig. 3 Patients with dactylitis at baseline (a), response at W12 (b), and resolution at W24 (c)

observed (data not shown). CZP does not have an Fc region and thus it does not activate antibody-dependent cell-mediated or complement-dependent cytotoxicity [14]. Therefore, CZP treatment should be taken into
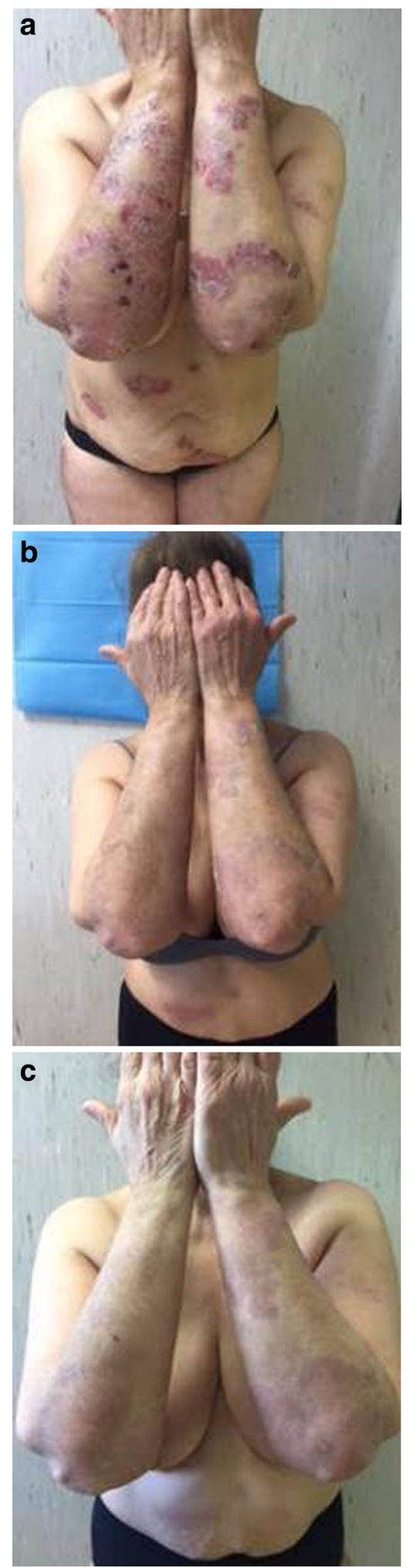

Fig. 4 Patients with psoriatic plaque at BL (a), result after treatment at W12 (b), and result at W24 (c) 

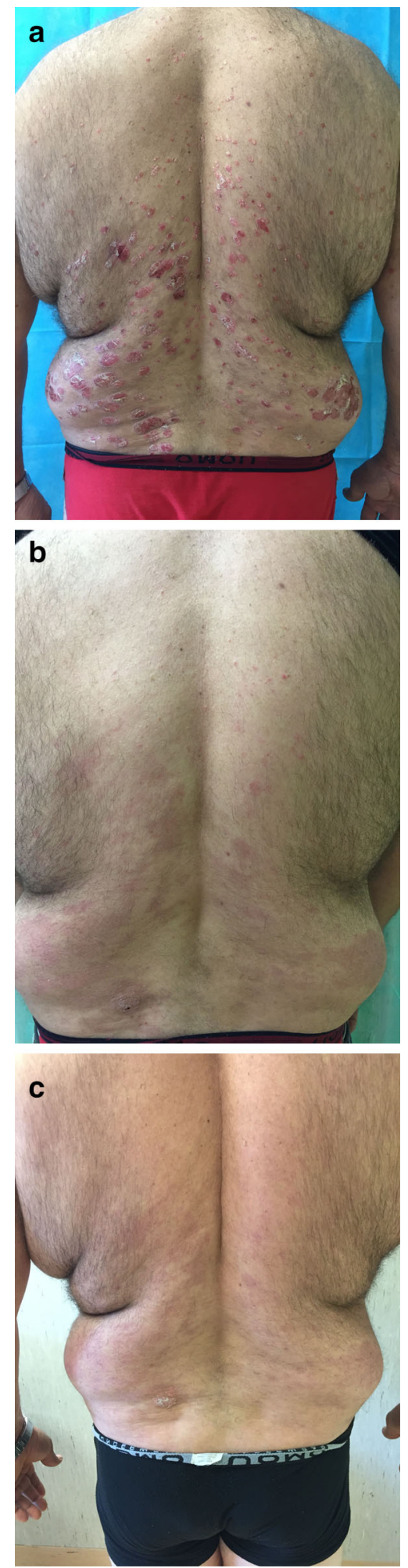

Fig. 5 Patients with psoriatic plaque on the trunk at $\mathrm{BL}$ (a), result after treatment at W12 (b), and result at W24 (c) consideration not only in patients unresponsive to synthetic DMARDs but also in those patients who have failed to respond to previous anti-TNF treatments, as has been demonstrated in several clinical trials and confirmed in our experience

Notably, due to the lack of a Fc region, CZP might also be a valid therapeutic option during pregnancy. In contrast to other whole antibody anti-TNFs, CZP crosses the placenta only by passive diffusion and could therefore be considered as the first line choice of treatment for the women with childbearing potential.

\section{Limitations of the study}

Although our experience is based on a small cohort of patients, evaluated over a short period of time, our data may be an important contribution to scientific knowledge. However, further analysis based on a larger cohort of patients and a longer period of treatment are required to answer the patients' biggest concern, namely, whether CZP is an effective and safe drug.

\section{CONCLUSIONS}

To the best of our knowledge our study is the first to demonstrate that CZP, in monotherapy or in combination therapy, may represent a valid, effective and well-tolerated therapeutic alternative for patients who have previously been exposed to other anti-TNF therapy in both joint and skin components.

\section{ACKNOWLEDGEMENTS}

No funding or sponsorship was received for this study or for the publication of this article. The article processing charges were funded by the authors. All named authors meet the International Committee of Medical Journal Editors (ICMJE) criteria for authorship for this manuscript, take responsibility for the integrity of the work as a whole, and have given final approval for the version to be published. 
Disclosures. Luca Bianchi served as speaker for Abbvie, Celegne, Jansen, Pfizer, Novartis, and UCB pharma. Annunziata Dattola, Maria Vittoria Cannizzaro, and Mauro Mazzeo have nothing to disclose.

Compliance with Ethics Guidelines. This study was conducted at the Department of Dermatology at the University of Rome "Tor Vergata". The study was approved by the local ethics committee in accordance with the ethical principles of the Declaration of Helsinki and was consistent with the guidelines for good clinical practice. All patients provided written consent.

Open Access. This article is distributed under the terms of the Creative Commons Attribution-NonCommercial 4.0 International License (http://creativecommons.org/licenses/ by-nc/4.0/), which permits any noncommercial use, distribution, and reproduction in any medium, provided you give appropriate credit to the original author(s) and the source, provide a link to the Creative Commons license, and indicate if changes were made.

\section{REFERENCES}

1. Prinz JC. The role of T cells in psoriasis. J Eur Acad Dermatol Venereol. 2003;17:257-70.

2. Christophers E. Psoriasis-epidemiology and clinical spectrum. Clin Exp Dermatol. 2001;26:314-20.

3. Kane D, Stafford L, Bresnihan B, FitzGerald O. A prospective, clinical and radiological study of early psoriatic arthritis: an early synovitis clinic experience. Rheumatology (Oxford). 2003;42:1460-8.

4. Lowes MA, Bowcock AM, Krueger JG. Pathogenesis and therapy of psoriasis. Nature. 2007;445:866-73.

5. Gerhard N, Krenn V, Magalhães R, Morawietz L, Brändlein S, König A. IgVH-genes analysis from psoriatic arthritis shows involvement of antigen-activated synovial B-lymphocytes. Z Rheumatol. $2002 ; 61(6): 718-27$.
6. Delgado Frias E, Diaz Gonzalez JF. Certolizumab pegol. Rheumatol Clin. 2011;6S3:S7-11. In Spanish.

7. Chimenti MS, Saraceno R, Chiricozzi A, Giunta A, Chimenti S, Perricone R. Profile of certolizumab and its potential in the treatment of psoriatic arthritis. Drug Des Dev Ther. 2013;7:339-48.

8. Smolen JS, et al. EULAR recommendations for the management of rheumatoid arthritis with synthetic and biological disease-modifying antirheumatic drugs: 2016 update. Ann Rheum Dis. 2017;76:960. https://doi.org/10.1136/annrheumdis-2016210715.

9. Gladman D, Fleischmann R, Coteur G, Woltering F, Mease PJ. Effect of certolizumab pegol on multiple facets of psoriatic arthritis as reported by patients: 24-week patient-reported outcome results of a phase III, multicenter study. Arthritis Care Res (Hoboken). 2014;66(7):1085-92. https://doi.org/10. $1002 /$ acr.22256.

10. Mease P. A short history of biological therapy for psoriatic arthritis. Clin Exp Rheumatol. 2015;33[5 Suppl 93]:S104-8.

11. Cauli A, Piga M, Lubrano E, Marchesoni A, Floris A, Mathieu A. New approaches in tumor necrosis factor antagonism for the treatment of psoriatic arthritis: certolizumab pegol. J Rheumatol Suppl. 2015;93:70-2. https://doi.org/10.3899/jrheum. 150641 .

12. Mease P, Deodhar A, Fleischmann R, Wollenhaupt J, Gladman D, Leszczyński P, Vitek P, Turkiewicz A, Khraishi M, FitzGerald O, Landewé R, de Longueville M, Hoepken B, Peterson L, van der Heijde D. Effect of certolizumab pegol over 96 weeks in patients with psoriatic arthritis with and without prior antitumour necrosis factor exposure. RMD Open. 2015;1(1):e000119. https://doi.org/10.1136/ rmdopen-2015-000119.

13. Gladman D, Fleischmann R, Szegvari B, Peterson L, Mease PJ. Long-term maintenance of improvements in multiple facets of psoriatic arthritis with certolizumab pegol: 96-week patient-reported outcome results of the rapid-Psa study. Value Health. 2014;17:A386.

14. Porter C, Armstrong-Fisher S, Kopotsha T, Smith B, Baker T, Kevorkian L, Nesbitt A. Certolizumab pegol does not bind the neonatal Fc receptor (FcRn): Consequences for FcRn-mediated in vitro transcytosis and ex vivo human placental transfer. J Reprod Immunol. 2016;116:7-12. https://doi.org/10. 1016/j.jri.2016.04.284 (epub 2016 Apr 14). 